\title{
An assessment of self-care knowledge among patients with diabetes mellitus
}

\begin{abstract}
Purposes: This study aimed to (1) assess the level of diabetes self-care knowledge among patients with diabetes mellitus and (2) examine the relationship between patients' diabetes selfcare knowledge and their demographic and medical characteristics.

Methods: A cross-sectional design was used to implement the study. A convenience sample of 273 diabetic patients were recruited from five primary health-care centers in Amman- Jordan.

Results: The overall level of knowledge of diabetes self-care in the total sample was moderate $(58.28 \%$ (SD $=18.24)$ ). The highest level of knowledge was meal planning $(70.2 \%)$ followed by monitoring, causes of diabetes, foot care, symptoms and complication, diabetic medication, and the lowest level was exercise (42.5\%). Furthermore, knowledge of diabetes self-care was found to be associated with age, educational status, diabetic medications and years with diabetes.

Conclusion: and Practice implications: The study findings emphasized that diabetic patients had a moderate level of knowledge and there were many of the learning needs for each area of knowledge. The health-care professional has an important role in developing the appropriate diabetes educational programs based on patients' learning needs and patients' characteristics. These programs that enhances knowledge on diabetes could be reduced or prevented diabetesrelated complications.
\end{abstract}

Keyword: Diabetes self-care knowledge; Patients with diabetes mellitus; A cross-sectional design 